\title{
Fast Multipole Representation of Green's Function for an Impedance Half-Space
}

\author{
Kamal Sarabandi, Fellow, IEEE, and Il-Suek Koh
}

\begin{abstract}
In this paper, exact and approximate multipole representations for fields of a horizontal or a vertical infinitesimal dipole above impedance surfaces are derived from the exact image formulation. These multipole expansions are appropriate for applications in the method of moments through a formulation known as the fast multipole method. Unlike the existing approximate formulations, these new multipole representations of the Green's function for the half-space impedance surface problem do not impose any restrictions on the location of source or observation points and are computationally very efficient.
\end{abstract}

Index Terms-Green's function, method of moments (MoM), multipole half-space.

\section{INTRODUCTION}

$\mathbf{T}$ HE SOMMERFELD problem of radiation of a short dipole above an infinite dielectric half-space is a classic problem in electromagnetics that has received significant attention. However, because of its relevance in a wide range of applications, it is still being examined. The formal solution is represented by integrals whose integrands are highly oscillatory and slowly decaying. These properties make direct numerical integration computationally inefficient. For impedance surfaces, however, an efficient method known as exact image theory has been implemented [1]. Since this formula replaces the poorly converging integrands of the Sommerfeld integral with exponentially decaying integrands, calculation of the fields from an arbitrary source above an impedance surface can be performed very efficiently, independent of the relative locations of the source and observation points. Hence it is now practical to apply this exact image representation as the half-space Green's function in integral equation (IE) numerical methods such as the method of moments (MoM). However, the numerical complexity of MoM is at best $O\left(n^{2}\right)$, which prohibits the application of MoM to electrically large objects.

In recent years, several methods have been proposed to overcome this difficulty. These include the fast multipole method (FMM) [2], adaptive integral method (AIM) [3], and many other variations of FMM and AIM. FMM has been rigorously formulated for the free-space Green's function and successfully applied to a large class of problems ranging from passive circuit analysis to computation of the radar cross-section (RCS)

\footnotetext{
Manuscript received July 31, 2002; revised February 26, 2003. This work was supported in part by the National Science Foundation under Contract ECS9979376 and in part by the PET program of the Department of Defense High Performance Computing Modernization Office.

The authors are with the Radiation Laboratory, Department of Electrical Engineering and Computer Science, The University of Michigan, Ann Arbor, MI 48109-2122 USA (saraband@eecs.umich.edu).

Digital Object Identifier 10.1109/TAP.2003.822440
}

of complex objects. In recent years, the FMM algorithm has been extended to include scattering problems in the presence of a dielectric half-space using the method of complex images [4]. The method of complex images uses Prony's method to approximate a component of the integrand of the Sommerfeld integral in terms of a summation of exponential functions, which can then be integrated analytically. The result is expressed as a summation of discrete image points in the complex $Z$ plane. The drawback of this method is that, depending upon the location of observation and source points (especially if they are close to the surface), the number of exponential terms in Prony's approximation has to be increased.

In this paper, by applying the exact image formulation, an efficient multipole representation of the Green's function for impedance surfaces is obtained and is shown to be very accurate for all locations of source and observation points. This paper is organized as follows: In Section II, the multipole representation is formulated. In Section III, the convergence property of the formulation is investigated and the accuracy of the results verified by comparing them with those obtained from direct numerical integration of the exact images.

\section{FASt Multipole RePRESENTATION OF GREEN's FunCtion FOR IMPEDANCE SURFACES}

The geometry of an elementary current source above an impedance surface is shown in Fig. 1. An infinitesimal dipole is oriented along unit vector $\hat{l}$ and located at $\left(x^{\prime}, y^{\prime}, z^{\prime}\right)$ above a surface with a normalized impedance of $\eta=Z_{1} / Z_{0}$. The observation point is located at $(x, y, z)$. A closed-form expansion for the total fields in the presence of the impedance surface using the exact image theory is given by [1]

$$
\begin{aligned}
\overrightarrow{\mathbf{E}} & =\overrightarrow{\mathbf{E}}^{d}+\overrightarrow{\mathbf{E}}^{i}+i 2 k_{0} Z_{0}\left[\frac{e^{i k_{0} R}}{4 \pi R}-\alpha \int_{0}^{\infty} e^{-\alpha \xi} \frac{e^{i k_{0} R^{\prime}}}{4 \pi R^{\prime}} d \xi\right] \\
& \times\left(l_{x} \hat{x}+l_{y} \hat{y}\right)-i 2 Z_{0} \eta l_{z} \hat{z} \\
& \times\left[\left(i \frac{\partial}{\partial z}+\beta\right) \frac{e^{i k_{0} R}}{4 \pi R}+\left(k_{0}^{2}-\beta^{2}\right) \int_{0}^{\infty} e^{-\beta \xi} \frac{e^{i k_{0} R^{\prime}}}{4 \pi R^{\prime}} d \xi\right] \\
& +2 Z_{0} \eta \hat{l} \times\left(\frac{\partial}{\partial y} \hat{x}-\frac{\partial}{\partial x} \hat{y}\right)\left[\frac{e^{i k_{0} R}}{4 \pi R}-\beta \int_{0}^{\infty} e^{-\beta \xi} \frac{e^{i k_{0} R^{\prime}}}{4 \pi R^{\prime}} d \xi\right] \\
& +i \frac{2 Z_{0} \eta}{1-\eta^{2}}\left\{\frac{\partial^{2}}{\partial x^{2}} l_{x} \hat{x}+\frac{\partial^{2}}{\partial y^{2}} l_{y} \hat{y}+\frac{\partial^{2}}{\partial x \partial y}\left(l_{y} \hat{x}+l_{x} \hat{y}\right)\right\} \\
& \times \int_{0}^{\infty}\left(e^{-\alpha \xi}-\eta^{2} e^{-\beta \xi}\right) \frac{e^{i k_{0} R^{\prime}}}{4 \pi R^{\prime}} d \xi .
\end{aligned}
$$

Here $\overrightarrow{\mathbf{E}}^{d}$ and $\overrightarrow{\mathbf{E}}^{i}$ are the direct dipole fields and the fields from its image if the surface is a perfect electric conductor, respectively. 


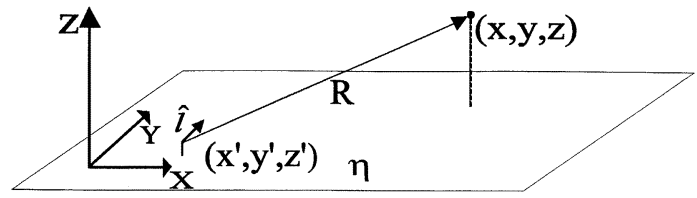

Fig. 1. Arbitrarily oriented infinitesimal dipole locates at $\left(x^{\prime}, y^{\prime}, z^{\prime}\right)$ over an impedance surface with a normalized impedance $\eta=Z_{0} / Z_{1}$.

Also in (1), $\alpha=k_{0} / \eta, \beta=k_{0} \eta, k_{0}$ is free-space propagation constant, $R^{\prime}=\sqrt{\left(x-x^{\prime}\right)^{2}+\left(y-y^{\prime}\right)^{2}+\left(z+z^{\prime}+i \xi\right)^{2}}$, $R=\sqrt{\left(x-x^{\prime}\right)^{2}+\left(y-y^{\prime}\right)^{2}+\left(z+z^{\prime}\right)^{2}}$, and $\hat{l}=l_{x} \hat{x}+$ $l_{y} \hat{y}+l_{z} \hat{z}$. Before proceeding with the development of the FMM representation of the total field (Green's function), note that all integrands in (1) contain two rapidly decaying exponential terms: one containing either $\alpha$ or $\beta$ and the other containing $R^{\prime}$, which has a positive imaginary part. These exponential decaying terms ensure fast convergence of Green's function for half-space impedance problems. Physically, $e^{-\alpha \xi}$ or $e^{-\beta \xi}$ can be interpreted as distributed image current in the complex $Z$ plane $(Z=z+i \xi)$. For inductive surfaces such as the earth, $|\eta|<1$, the decay factor for the horizontal polarization is much higher than the vertical polarization. In extreme cases where $|\eta| \ll 1$, the decay factor of the integrand for the vertical polarization is dominated by $R^{\prime}$, as mentioned earlier.

Multipole representation of dyadic Green's function for applications in integral equations method has been proven to be useful with regard to computational speed and resources. Examining (1), it is obvious that the FMM algorithms [2] can be used for $\overrightarrow{\mathbf{E}}^{d}$ and $\overrightarrow{\mathbf{E}}^{i}$ directly. However, for the rest of expressions in (1), an additional multipole representation is needed in order to decompose the Green's function in terms of a summation of the products of two functions: one dependent on the source coordinate and the other dependent on the observation coordinate. To achieve this goal, a multipole expansion for $I=\int_{0}^{\infty} e^{-p \xi}\left(e^{i k_{0} R^{\prime}} / R^{\prime}\right) d \xi$ is needed where $p$ represents $\alpha$ or $\beta$. One can attempt to express $e^{i k_{0} R^{\prime}} / R^{\prime}$ in the integrand of $I$ in terms of its multipole expansion. However, since the domain of $\xi$ extends to infinity, such substitution cannot be rigorously justified despite the fact that, due to the exponential decay, the integral does not contribute significantly for large values of $\xi$. We will revisit this approach after deriving a formal solution.

To derive a formal multipole expansion for the Green's function, a new integral expression for $I$ is obtained. Integrating $I$ by parts and noting $(\partial / \partial \xi)\left(e^{i k_{0} R^{\prime}} / R^{\prime}\right)=i\left(\partial / \partial z^{\prime}\right)\left(e^{i k_{0} R^{\prime}} / R^{\prime}\right)$, it can be shown that $I$ must satisfy the following first-order differential equation:

$$
\frac{\partial I}{\partial z^{\prime}}+i p I=i \frac{e^{i k_{0} R}}{R}
$$

The general solution of (2) for $I$ is composed of the particular solution and the homogeneous solution and given by

$$
I=i e^{-i p z^{\prime}} \int \frac{e^{i k_{0} R}}{R} e^{i p z^{\prime}} d z^{\prime}+C e^{-i p z^{\prime}}
$$

where $C$ is a constant function of $z^{\prime}$. Note that new integral representation is highly oscillatory and not amenable for numerical evaluation. However, as will be shown next, this expression is

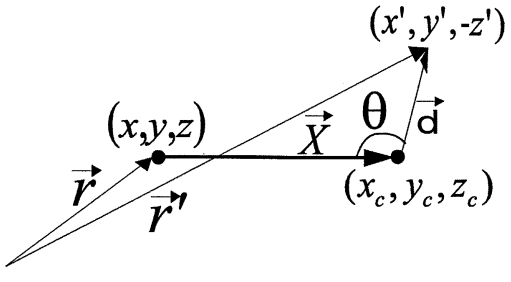

Fig. 2. Definition of vectors $\vec{X}$ and $\vec{d}$ in the formulation of multipole representation of (4).

suitable for multipole expansions. But first the coefficient $C$ in (3) must be determined.

To find $C$, it is noted that $\lim _{z^{\prime} \rightarrow \infty} e^{-i p z^{\prime}}=\infty$ in the upper half-plane of the $p$ plane, where $\Im[p]>0$. However, since $I$ must approach zero due to the radiation condition, $C$ must be zero when $\Im[p]>0$. Noting that $I$ is an analytic function of $p$, then according to analytic continuation, $C$ must be zero for all values of $p$ (the entire plane). Now a multipole representation of $I$ can be obtained using the Gegenbauer's addition theorem. According to Fig. 2

$$
\frac{e^{i k_{0} R}}{R}=\frac{e^{i k_{0}\left|\vec{r}-\overrightarrow{r^{\prime}}\right|}}{\left|\vec{r}-\overrightarrow{r^{\prime}}\right|}=\frac{e^{i k_{0}|\vec{X}+\vec{d}|}}{|\vec{X}+\vec{d}|}
$$

Requiring $|\vec{d}|<|\vec{X}|$, Gegenbauer's addition theorem [5] states that

$$
\frac{e^{i k_{0} R}}{R}=i k_{0} \sum_{n=0}^{\infty}(-1)^{n}(2 n+1) P_{n}(\cos \theta) h_{n}\left(k_{0} X\right) j_{n}\left(k_{0} d\right)
$$

where $d=|\vec{d}|=\sqrt{\left(x^{\prime}-x_{c}\right)^{2}+\left(y^{\prime}-y_{c}\right)^{2}+\left(z^{\prime}-z_{c}\right)^{2}}$, $X=|\vec{X}|=\sqrt{\left(x_{c}-x\right)^{2}+\left(y_{c}-y\right)^{2}+\left(z_{c}+z\right)^{2}}$, and $\theta$ is the angle between the $\vec{X}$ and $\vec{d}$ vectors given by $\theta=\cos ^{-1}(\vec{X} \cdot \vec{d})$, as shown in Fig. 2. Also here, $P_{n}$ is the Legendre polynomial and $h_{n}$ and $j_{n}$ are spherical Bessel functions of $n$th order. The plane wave expansion representation of $P_{n} j_{n}$ is given by [2]

$$
P_{n}(\cos \theta) j_{n}\left(k_{0} d\right)=\frac{(-i)^{n}}{4 \pi} \int_{4 \pi} d^{2} \hat{k} e^{i \vec{k} \cdot \vec{d}} P_{n}(\hat{k} \cdot \hat{X})
$$

where $\vec{k}=k_{0}(\sin \nu \cos \psi \hat{x}+\sin \nu \sin \psi \hat{y}+\cos \nu \hat{z})$ and $\hat{k}=$ $\vec{k} / k_{0}$. Substituting (5) in (4) and then performing integration with respect to $z^{\prime}, I$ can be written as

$$
\begin{aligned}
I=\frac{i k_{0}}{4 \pi} \sum_{n=0}^{\infty} i^{n}(2 n+1) & h_{n}\left(k_{0} X\right) \\
& \times \int_{4 \pi} d^{2} \hat{k} P_{n}(\hat{k} \cdot \hat{X}) \frac{e^{i \vec{k} \cdot \vec{d}}}{k_{0} \cos \nu+p} .
\end{aligned}
$$

This formal solution, within the radius of convergence of the series, is an exact representation of the multipole expansion for the Green's function, which will be verified numerically. Also the regions of convergence of the series will be identified. It is important to note that (6), except for the factor $1 /\left(k_{0} \cos \nu+p\right)$, is identical to that of the free-space multipole representation of the Green's function, and therefore is of the same computational complexity. As will be shown later, (6) converges very quickly when $|p / k|>1$, which is the case for horizontal polarization 


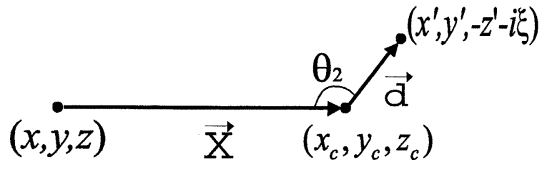

Fig. 3. Definition of vectors $\vec{X}$ and $\vec{d}$ in the formulation of multipole representation of (8).

( $\left.p=\alpha=k_{0} / \eta\right)$ and when the magnitude of the normalized surface impedance is less than unity $|\eta|<1$. These values of normalized surface impedance correspond to ground impedances at ultra-high-frequency band and below, or water surfaces below $10 \mathrm{GHz}$.

Unfortunately, the series convergence may become rather poor for vertical polarization when $p=\beta$. As $\Re[p]$ decreases, each term in the summation decays slowly with $n$, and the partial summation starts to oscillate. This increases the required number of terms in the summation for convergence, and hence the expansion becomes numerically unstable. The formal derivation of (6) seems to be exact, and therefore the lack of convergence of the series under certain conditions for the vertical polarization must be further examined. This problem can be a result of interchanging the order of the infinite summation and the integration. This interchange is not always allowed according to Lebesque's dominated convergence theorem.

To verify the validity of (6) (where the series converges), another independent approach may be followed. Noting the exponential decays in the integrand of $I$, the integral range can be truncated, i.e.,

$$
I=\int_{0}^{\infty} e^{-p \xi} \frac{e^{i k_{0} R^{\prime}}}{R^{\prime}} d \xi \approx \int_{0}^{a} e^{-p \xi} \frac{e^{i k_{0} R^{\prime}}}{R^{\prime}} d \xi
$$

where $a$ is chosen to satisfy $\left.e^{\Re\left[i k_{0} R^{\prime}-p \xi\right]}\right|_{\xi=a} \ll 1$. This choice of $a$ also satisfies the condition for which the following addition theorem for the scalar Green's function is valid. Using Gegenbauer's addition theorem for complex quantities, the integrand of (7) (scalar Green's function with complex range function) is expanded as

$$
\frac{e^{i k_{0} R^{\prime}}}{R^{\prime}}=i k_{0} \sum_{n=0}^{\infty}(-1)^{n}(2 n+1) P_{n}\left(\cos \theta_{2}\right) h_{n}\left(k_{0} X\right) j_{n}\left(k_{0} d\right)
$$

where $X=\sqrt{\left(x_{c}-x\right)^{2}+\left(y_{c}-y\right)^{2}+\left(z_{c}+z\right)^{2}}, d=$ $\sqrt{\left(x^{\prime}-x_{c}\right)^{2}+\left(y^{\prime}-y_{c}\right)^{2}+\left(z^{\prime}+z_{c}+i \xi\right)^{2}}$, and $\theta_{2}$ is a complex angle between $\vec{X}$ and $\vec{d}$ vectors given by $\theta_{2}=\cos ^{-1}(\vec{X} \cdot \vec{d})$ (see Fig. 3). This expansion is valid for the condition $|X|>\left|d e^{ \pm i \theta}\right|$. Using the identity given by (5)

$$
\begin{aligned}
I \approx \frac{i k_{0}}{4 \pi} \sum_{n=0}^{\infty} i^{n}(2 n+1) h_{n}\left(k_{0} X\right) \\
\quad \times \int_{4 \pi} d^{2} \hat{k} P_{n}(\hat{k} \cdot \hat{X}) e^{i \vec{k} \cdot \vec{d}} \frac{1-e^{-\left(k_{0} \cos \nu+p\right) a}}{k_{0} \cos \nu+p} .
\end{aligned}
$$

In $\lim _{a \rightarrow \infty}$, the exponential function in the integrand of (9) disappears, and the expression reduces to the formal solution previously derived. Therefore, the formal solution converges for a large value of $\Re[p]$ but the convergence rate becomes poorer when $\Re[p]$ decreases.

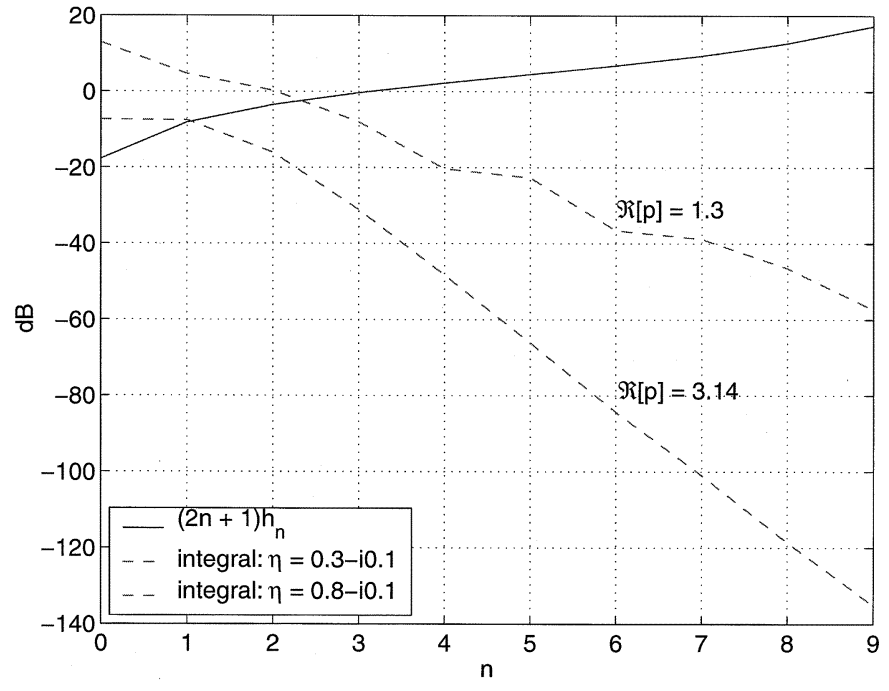

Fig. 4. Convergence behavior of (6) as a function of $\Re[p]$. Magnitude of each term of the summation is shown.

For $p=\beta$ and in situations when convergence is poor (the region of poor convergence which will be specified later), an approximate identity can be formulated. However, this approximate formulation is much less efficient than the expansion given by (6). Applying a Gaussian quadrature numerical integration applied to the exact image representation, $I$ can be approximated as a finite summation of the form that resembles the method of complex images, i.e.

$$
I \approx \sum_{n=1}^{N} w_{n} e^{-p \xi_{n}} \frac{e^{i k_{0} R_{n}^{\prime}}}{R_{n}^{\prime}}
$$

where $R_{n}^{\prime}=\sqrt{\left(x-x^{\prime}\right)^{2}+\left(y-y^{\prime}\right)^{2}+\left(z+z^{\prime}+i \xi_{n}\right)^{2}}$ with $w_{n}$ and $\xi_{n}$ being the weighting factor and zero of the Legendre polynomial of $n$th order. The discrete terms $e^{i k_{0} R_{n}^{\prime}} / R_{n}^{\prime}$ in (10) can be expanded using (8). Because of the exponential decay in the integrand $\left(e^{-p \xi}\right)$, the order $N$ can be chosen so that the accuracy of (10) is independent of source and observation coordinates.

\section{Simulation Results}

For a frequency of $50 \mathrm{MHz}$ and a surface with a normalized surface impedance $\eta=0.3-i 0.1(\alpha=3.14+1.05 i$ and $\beta=0.31-0.11 i$ ), the FMM expressions for $I$ are compared with the direct numerical calculations. For simplicity, $(x, y, z)$ is located at $(0,0,0)$ and $\left(x_{c}, y_{c}, z_{c}\right)$ is at $(7,0,2.2)$. For numerical simulations, the following identity is used, which can be derived directly from (6):

$$
\begin{aligned}
\frac{e^{i k_{0} R}}{4 \pi R} & -p \int_{0}^{\infty} e^{-p \xi} \frac{e^{i k_{0} R^{\prime}}}{4 \pi R^{\prime}} d \xi=\frac{i k_{0}}{(4 \pi)^{2}} \sum_{n=0}^{\infty} i^{n}(2 n+1) h_{n} \\
& \times\left(k_{0} X\right) \int_{0}^{2 \pi} \int_{0}^{\pi} P_{n}(\hat{k} \cdot \hat{X}) \frac{k_{0} e^{i \vec{k} \cdot \vec{d}} \cos \nu}{k_{0} \cos \nu+p} \sin \nu d \nu d \psi .
\end{aligned}
$$

Fig. 4 shows the magnitude of each term of the summation given by (11) for $p=\alpha$ as a function of $n$ for two different surface impedances $\eta=0.3-i 0.1 \quad(\Re[p]=3.14)$ and $\eta=$ $0.8-i 0.1(\Re[p]=1.3)$. In this figure, it is clearly shown that 


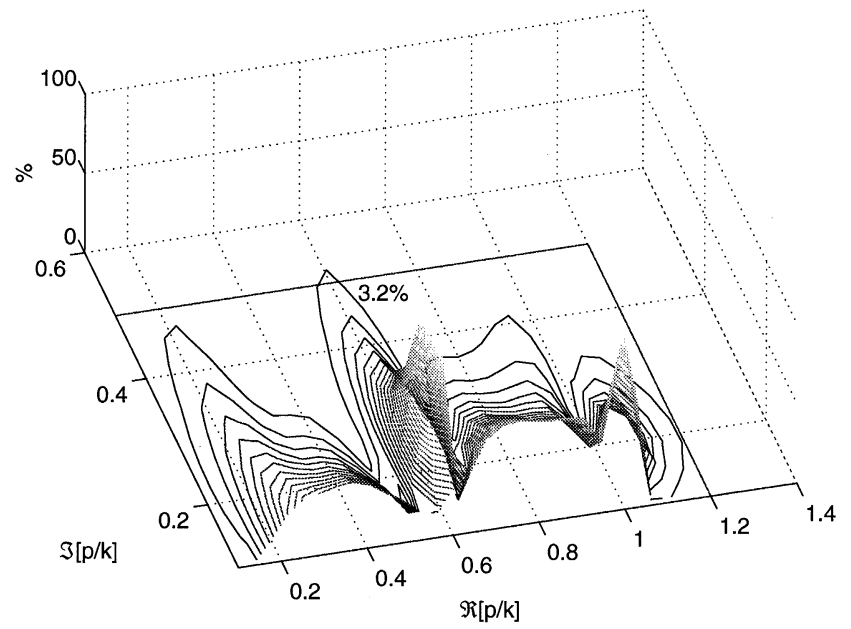

(a)

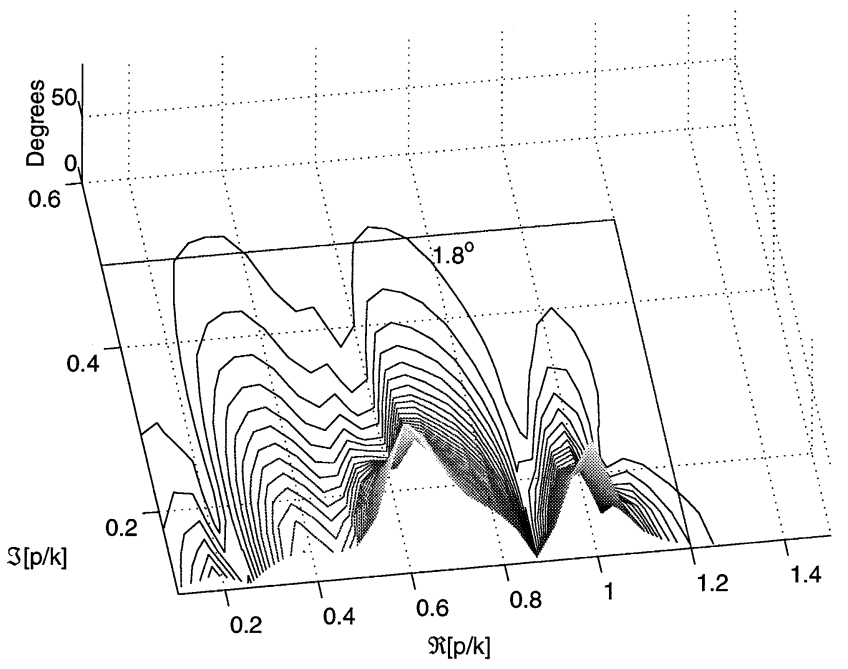

(b)

Fig. 5. Error between left-hand side (numerical integration) and right-hand side (FMM identity) of (11) as a function of $p / k$ at $50 \mathrm{MHz}$. Observation point is fixed at $(7 \cdot 5,0,2.2)$. (a) Percentage error of magnitude $(<100 \%)$ and (b) phase difference $\left(<90^{\circ}\right)$.

as the real part of $p$ decreases, the convergence of the summation becomes poorer (terms decay much slower), and the partial sum begins to oscillate as mentioned earlier. The following figures show comparisons of the results obtained from the FMM expression given by (6) and (10) with those obtained from direct numerical integration. Fig. 5 is a plot of the difference between the direct numerical integration [left-hand side of (11)] and its FMM representation [right-hand side of (11)] as a function of the real and imaginary part of $p / k$. For this calculation, observation and source points are, respectively, fixed at $(7.5,0,2.2)$ and $(0,0,0)$, and the number of summation terms $(N)$ is ten. As seen in this figure, except for the case of $\Im[p / k]<0.5$ and $\Re[p / k]<1.2$, the expansion given by (6) gives excellent results. Hence for all practical purposes, the identity given by (6) can be used except when $\Re[p / k]<1.2$ and $\Im[p / k]<0.5$. In this region $(\Re[p / k]<1.2$ and $\Im[p / k]<0.5)$, the less efficient formula given by (10) may be used. By changing the location of source and observation points, it was determined that the region of validity of (6) is independent of the coordinates of the source

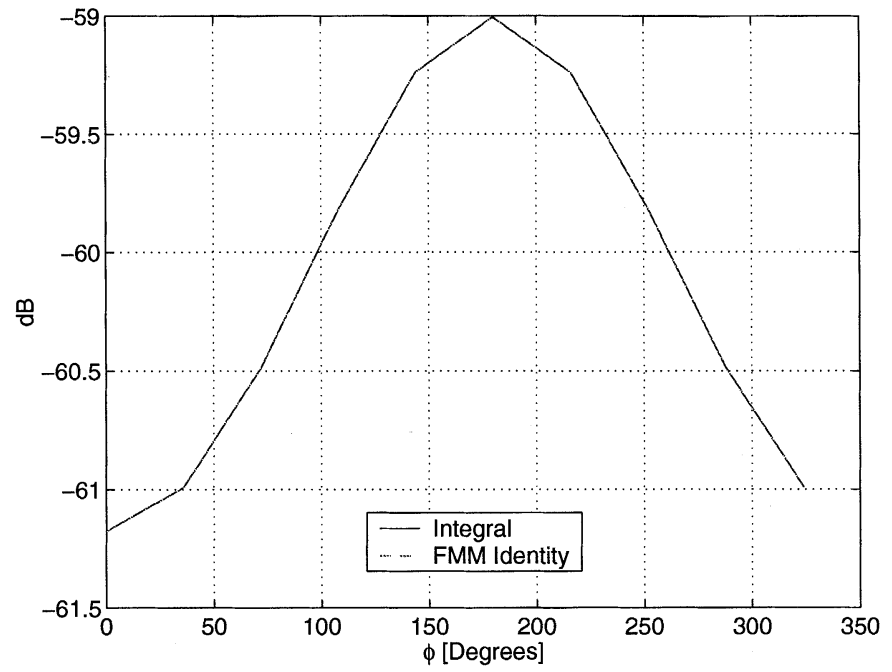

(a)

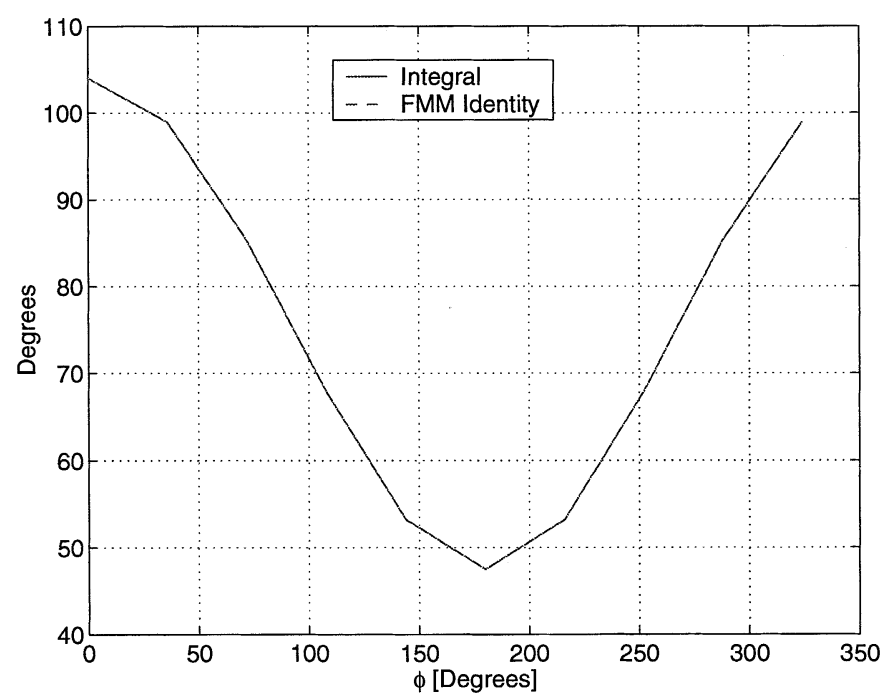

(b)

Fig. 6. Comparison of left-hand side (numerical integration) and right-hand side (FMM identity) of (11) when $p=\alpha=3.14+1.05 i$ as a function of observation point positions, $(7+0.5 \cos \phi, 0.5 \sin \phi, 2.2)$. (a) Magnitude and (b) phase.

and observation points. For a numerical comparison, an example is considered when the observation point is moved along a parameterized curve $x=x_{c}+0.5 \cos \phi, y=y_{c}+0.5 \sin \phi$, and $z=z_{c}$. Fig. 6(a) and (b) shows comparisons of the magnitude, and the phase of the left- and right-hand side of (11) for $p=\alpha=3.14+1.05 i$, respectively. These two results are in excellent agreement. Fig. 7(a) and (b) shows the same comparisons for $p=\beta=0.31-0.11 i$. For this case (choosing $N=10$ ), discrepancies of less than $0.2 \mathrm{~dB}$ in the magnitude and $1^{\circ}$ in phase are observed.

The next consideration is of convergence properties of the FMM formulation versus distance. Fig. 8 shows an example of this examination. For this figure, (11) is computed again along an observation line $(7+3 t \cos (2 \pi t), 3 t \sin (2 \pi t), 2.2)$, where $0 \leq t \leq 1$, for $p=\alpha=3.14+1.05 i$, and a fixed $N=10$. The compared results are in excellent agreement. By increasing $N$ to 20 , the results are not affected. A required number of terms for convergence of the FMM identity can 


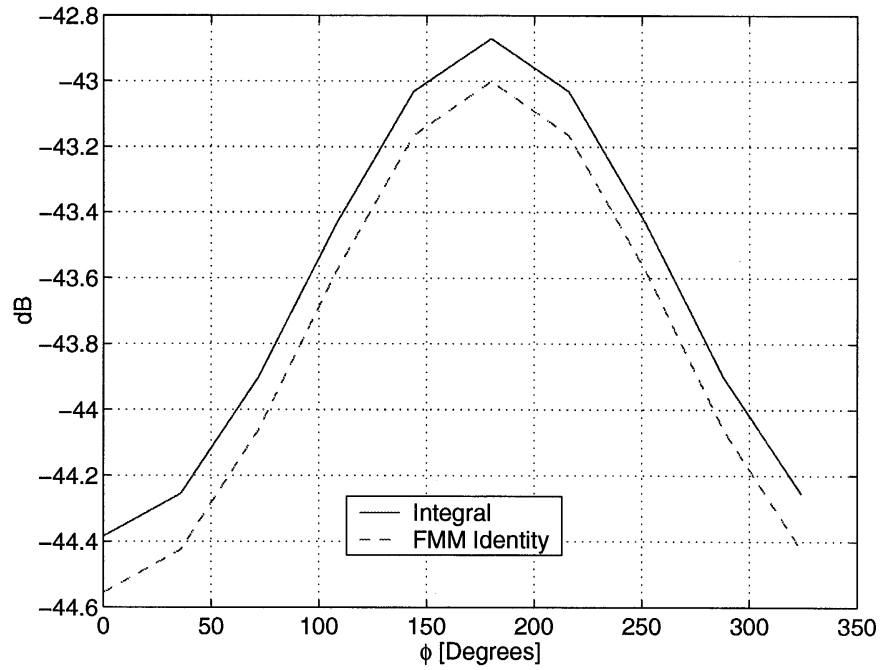

(a)

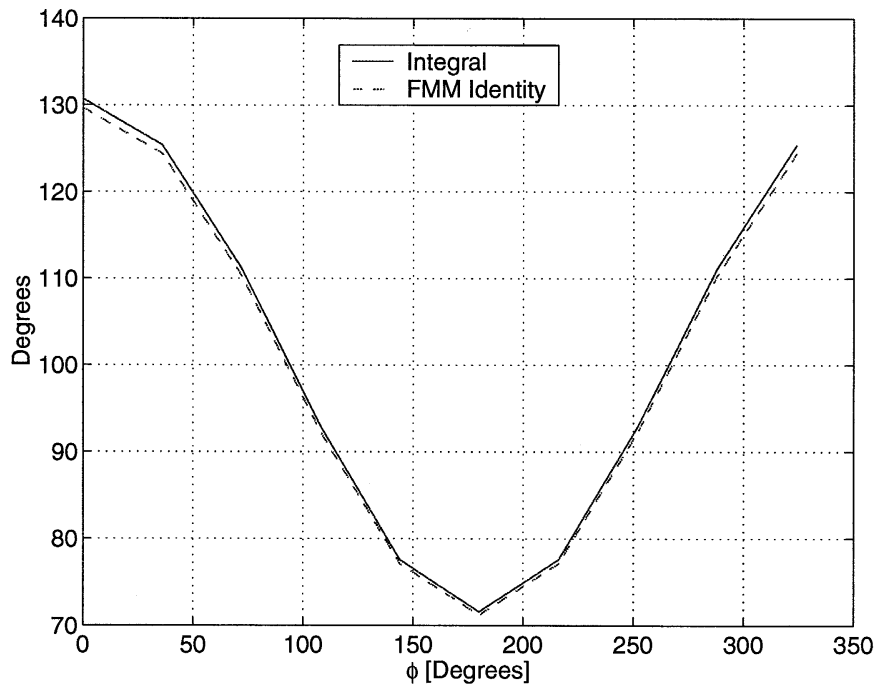

(b)

Fig. 7. Comparison of left-hand side (numerical integration) and right-hand side (FMM identity) of (11) when $p=\beta=0.31-0.11 i$ as a function of observation point positions, $(7+0.5 \cos \phi, 0.5 \sin \phi, 2.2)$. (a) Magnitude and (b) phase.

be estimated at approximately 12 for single precision, and 21 for double precision using the well-known semiempirical equation for the free-space case [2]. Therefore the FMM (11) has the same convergence property as that of the free-space one when in the region of convergence of the summation.

\section{CONCLUSION}

Two multipole representations of the field of a dipole above impedance surfaces are formulated. These formulations are suitable for FMM-type algorithms applied to scattering problems for an object over/on an impedance surface. One formulation is exact and valid for the range of $\Re[p / k]>1.2$ and $\Im[p / k]<0.5$ and the other, which is approximate and computationally less efficient, for the complement region. These representations are verified by comparing them with a direct numerical integration. It is shown that the exact multipole expansion for the Green's

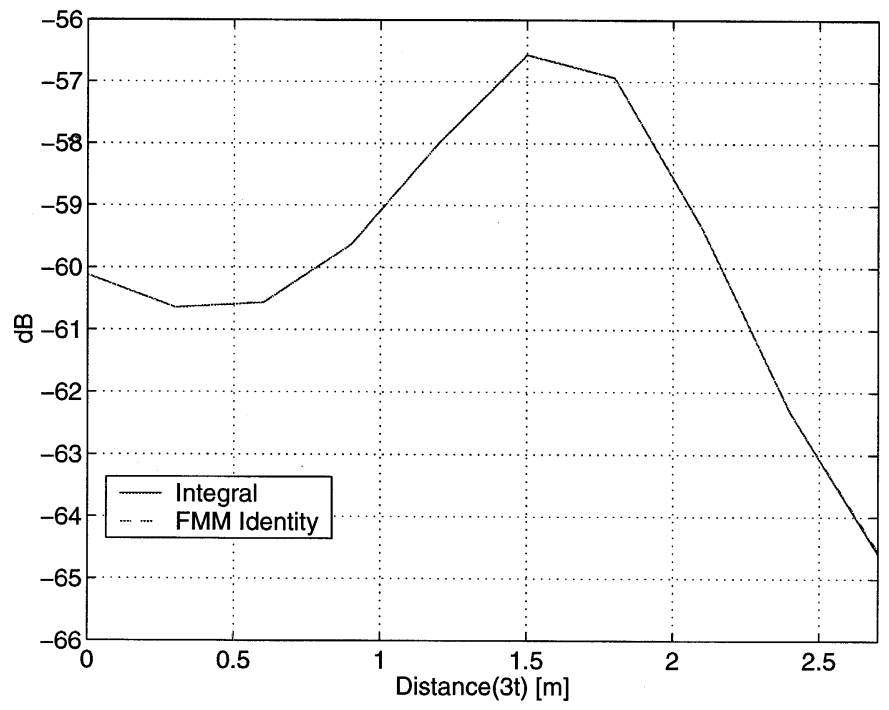

(a)

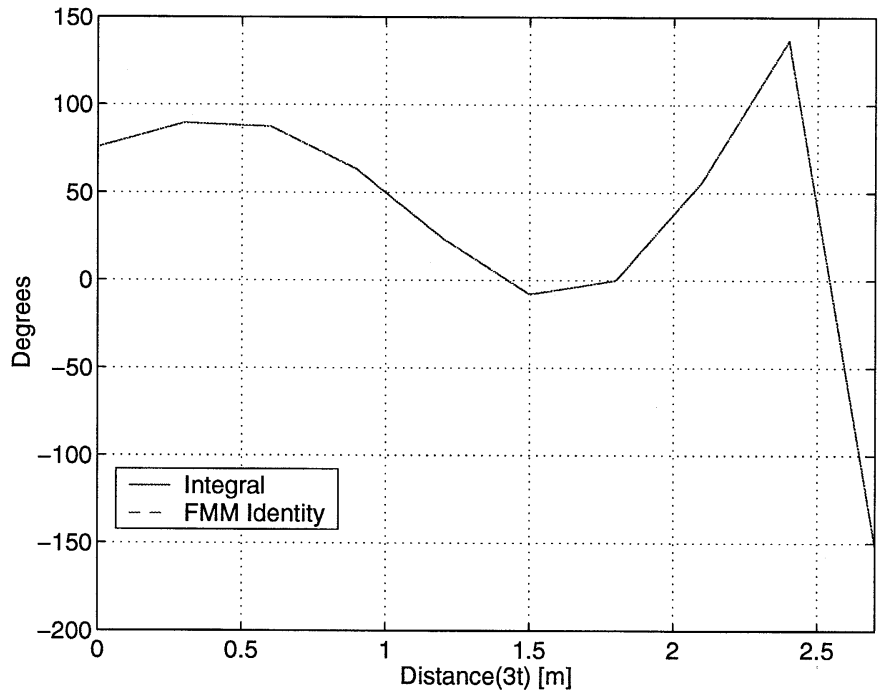

(b)

Fig. 8. Comparison of left-hand side (numerical integration) and right-hand side (FMM identity) of (11) when $p=\alpha=3.14+1.05 i$ as a function of observation point positions, $(7+3 t \cos (2 \pi t), 3 t \sin (2 \pi t), 2.2)$, where $0 \leq$ $t \leq 1$. (a) Magnitude and (b) phase.

function of the half-space problem is very similar to that for the free-space case, and hence has the same computational complexity and convergence property.

\section{REFERENCES}

[1] K. Sarabandi, M. D. Casciato, and I. Koh, "Efficient calculation of the field of a dipole radiating above an impedance surface," IEEE Trans. Antennas Propagat., vol. 50, pp. 1222-1235, Sept. 2002.

[2] R. Coifman, V. Rokhlin, and S. Wandzura, "The fast multipole method for the wave equation: A pedestrian prescription," IEEE Antennas Propagat. Mag., vol. 35, pp. 7-12, June 1993.

[3] E. Bleszynski, M. Bleszynski, and T. Jaroszewicz, "Aim: Adaptive integral method for solving large-scale electromagnetic scattering and radiation problems," Radio Sci., vol. 31, no. 5, pp. 1225-1251, Sept.-Oct. 1996.

[4] N. Geng, A. Sullivan, and L. Carin, "Fast multipole method for scattering form an arbitrary PEC target above or buried in a lossy half space," IEEE Trans. Antennas Propagat., vol. 49, pp. 740-748, May 2001.

[5] M. Abramowitz and I. A. Stegun, Handbook of Mathematical Functions with Formulas, Graphs, and Mathematical Tables. New York: Wiley, 1972. 


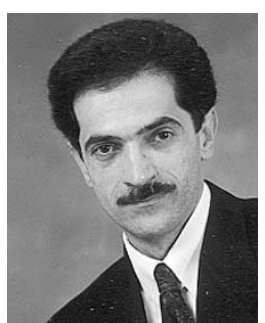

Kamal Sarabandi (S'87-M'90-SM'92-F'00) received the B.S. degree in electrical engineering from Sharif University of Technology, Tehran, Iran, in 1980. He received the M.S. degree in electrical engineering and in mathematics and the $\mathrm{Ph} . \mathrm{D}$. degree in electrical engineering from The University of Michigan, Ann Arbor, in 1986 and 1989, respectively.

$\mathrm{He}$ is Director of the Radiation Laboratory and a Professor in the Department of Electrical Engineering and Computer Science, The University of Michigan. His research areas of interest include microwave and millimeter-wave radar remote sensing, electromagnetic wave propagation, and antenna miniaturization. He has 20 years of experience with wave propagation in random media, communication channel modeling, microwave sensors, and radar systems and is leading a large research group. Over the past ten years he has graduated $20 \mathrm{Ph} . \mathrm{D}$. students. He has served as the Principal Investigator on many projects sponsored by NASA, JPL, ARO, ONR, ARL, NSF, DARPA, and numerous industries. He has published many book chapters and more than 105 papers in refereed journals on electromagnetic scattering, random media modeling, wave propagation, antennas, microwave measurement techniques, radar calibration, inverse scattering problems, and microwave sensors. He has had more than 220 papers and invited presentations in national and international conferences and symposia on similar subjects.

Dr. Sarabandi is a Member of URSI Commission F and of The Electromagnetic Academy. He is a Vice President of the IEEE Geoscience and Remote Sensing Society (GRSS), a past Chairman of the Awards Committee of the IEEE GRSS from 1998 to 2002, and a Member of the IEEE Technical Activities Board Awards Committee from 2000 to 2002. He is Associate Editor of the IEEE TRANSACTIONS ON ANTENNAS AND PROPAGATION (AP) and the IEEE SENSORS JOURNAL. He is listed in American Men \& Women of Science, Who's Who in America, and Who's Who in Electromagnetics. He received the Henry Russel Award from the Regent of The University of Michigan (the highest honor the University of Michigan bestows on a faculty member at the assistant or associate level). In 1999, he received a GAAC Distinguished Lecturer Award from the German Federal Ministry for Education, Science, and Technology. He also received a 1996 Teaching Excellence Award from the Department of Electrical Engineering and Computer Science, and the 2003-2004 College of Engineering Research Excellence Award, The University of Michigan.
Il-Suek Koh was born in Korea. He received the B.S. and M.S. degrees in electronics engineering from Yonsei University, Seoul, Korea, in 1992 and 1994, respectively, and the Ph.D. degree from The University of Michigan, Ann Arbor, in 2002 .

In 1994, he joined LG Electronics Ltd., Seoul, as a Research Engineer. Currently, he is with The University of Michigan as a Research Fellow. His research interests are theoretical modeling of wireless communication channel, multibody problems, and various numerical and analytical techniques. 